Anaesthesist 2020 $69: 453-462$

https://doi.org/10.1007/s00101-020-00770-1

Online publiziert: 28. April 2020

(c) Der/die Autor(en) 2020, korrigierte

Publikation 2020

\section{S. Koch ${ }^{1}$. S. Pecher ${ }^{2}$}

${ }^{1}$ Klinik für Anästhesiologie mit Schwerpunkt operative Intensivmedizin, Charité - Universitätsmedizin Berlin, Campus Virchow-Klinikum und Campus Mitte, Berlin, Deutschland

${ }^{2}$ Klinik für Anästhesie und Intensivmedizin, Diakonie Klinikum Stuttgart, Stuttgart, Deutschland

\title{
Neue Herausforderungen für die Anästhesie durch den Klimawandel
}

\begin{abstract}
Der Gesundheitssektor trägt mit 5-10\% zu den Treibhausgasemissionen in westlichen Ländern bei, wobei vor allem die energieintensiven $\mathbf{A b}$ teilungen (Intensivstationen/OP Bereich, über $\mathbf{5 0} \%$ der Treibhausgasemissionen eines Krankenhauses), sowie die Nutzung inhalativer Anästhetika ( 35\% der Treibhausgasemissionen eines Krankenhauses) einen großen Anteil haben [1-3]. Die gesundheitlichen Bedrohungen durch den Klimawandel entstehen durch häufigere Extremwetterereignisse, Umweltverschmutzung, die Ausbreitung von Tropenkrankheiten etc. [4]. Ein stabiles Klima ist für unsere Gesundheit und unser Überleben essentiell, als Ärzte sollten wir diese Herausforderungen annehmen und handeln.
\end{abstract}

\section{Hintergrund}

Die Erderwärmung ist bedingt durch einen dramatischen Anstieg der Treibhausgase, verursacht durch die Verbrennung fossiler Treibstoffe (Kohlendioxid $\left[\mathrm{CO}_{2}\right]$ ), durch Massentierhaltung, das Auftauen der Permafrostböden (Methan $\left.\left[\mathrm{CH}_{4}\right]\right)$ sowie einer nicht nachhaltigen Land- und Forstwirtschaft $\left(\mathrm{CH}_{4}\right.$ und Lachgas $\left[\mathrm{N}_{2} \mathrm{O}\right]$ ). Kohlendioxid, Methan und Lachgas gehören zu den natürlichen Treibhausgasen, während halogenierte Kohlenwasserstoffe künstliche Treibhausgase sind [5]. Treibhausgase entfalten ihre erderwärmende Wirkung durch Reflexion infraroter Wärmestrahlung von der Troposphäre zurück zur
Erde [6]. Die Energiebilanz der Erde wird von folgenden 3 Einflussgrößen bestimmt: 1) Intensität der einfallenden Sonnenstrahlung, 2) direkte Reflexion von Sonnenstrahlen durch Aerosole in der Atmosphäre und durch helle Eisflächen auf der Erdoberfläche (AlbedoEffekt) sowie 3) Abstrahlung infraroter Wärmestrahlung ins Weltall. Letzteres ist der wichtigste Mechanismus zur Abkühlung der Erde. Gleichzeitig ist dieser natürliche Treibhauseffekt für ein stabiles, lebenswertes Klima auf der Erde notwendig. Seit 800.000 Jahren befindet sich das Klimasystem der Erde in einem stabilen Bereich; erst dies ermöglichte die Entstehung des Homo sapiens (Beginn vor ca. 300.000 Jahren; [7]). Während dieser Zeitspanne lag die "physiologische" $\mathrm{CO}_{2}$ Konzentration zwischen 180-280 ppm (parts per million) - heute, aufgrund der ungebremsten Verbrennung fossiler Energieträger in den letzten 50 bis 80 Jahren, beträgt sie aktuell $413 \mathrm{ppm}$ [8] und bedingt eine globale Erwärmung von aktuell $1,1^{\circ} \mathrm{C}$ (• Abb. 1; [9]). Der anthropogene Einfluss hat die Treibhausgase auf - in den letzten 800.000 Jahren nie da gewesene Werte - ansteigen lassen, um $45 \%$ über normal für $\mathrm{CO}_{2}$, um $155 \%$ für $\mathrm{CH}_{4}$ und um $22 \%$ für $\mathrm{N}_{2} \mathrm{O}$ [9]. Das wichtige Gleichgewicht der Energiebilanz der Erde ist dramatisch aus der Balance geraten.

Das Paris Agreement hat zum Ziel, die Erderwärmung unter $1,5^{\circ} \mathrm{C}$ über dem vorindustriellen Level zu halten. Im Bericht des Weltklimarats 2018 wird das $1,5^{\circ} \mathrm{C}$-Ziel nochmals betont, da bei ei- ner weiteren Erwärmung nur noch eine $66 \%$ ige Chance besteht, die sog. Kippelemente im Klimasystem nicht zu überschreiten [11]. Kippelemente, wie beispielsweise das Abschmelzen der Polkappen und des Grönlandeises, der Verlust des Amazonas-Regenwalds, das Auftauen der Permafrostböden und vieles mehr, werden beim Überschreiten selbstverstärkende Feedbackschleifen und Dominoeffekte auslösen und zu einer nicht mehr zu beeinflussenden Klimaerwärmung führen, mit desaströsem Ausgang für die Menschheit. Der Weltklimarat 2018 hat daher nochmals eindringlich gefordert, die jährlichen Emissionen weltweit bis 2030 mindestens zu halbieren und bis 2050 eine Nettobilanz von Null $\mathrm{zu}$ erreichen. Die von Deutschland im Rahmen des Klimapakets im November 2019 verabschiedeten Ziele sind dafür nicht ausreichend und lassen die Welt auf eine Klimaerwärmung von $3-4^{\circ} \mathrm{C}$ zusteuern [12]. Gesundheitliche Bedrohungen durch den Klimawandel entstehen durch vermehrte Stürme, Dürren, Überschwemmungen und Hitzewellen. Indirekte Folgen sind nicht weniger gravierend: Krankheiten, die bisher eher in den Tropen verbreitet waren, wie Malaria, Dengue-Fieber und Cholera, werden auch in Mitteleuropa heimisch, Hunger durch Ernteausfälle, Wassermangel und Migration durch sich verringernde Lebensräume. All diese Einflüsse werden Konsequenzen auf die allgemeine und psychische Gesundheit haben; sie werden politische Systeme gefährden und könnten Kriege um die letzten Ressourcen der Erde entfachen [11]. 


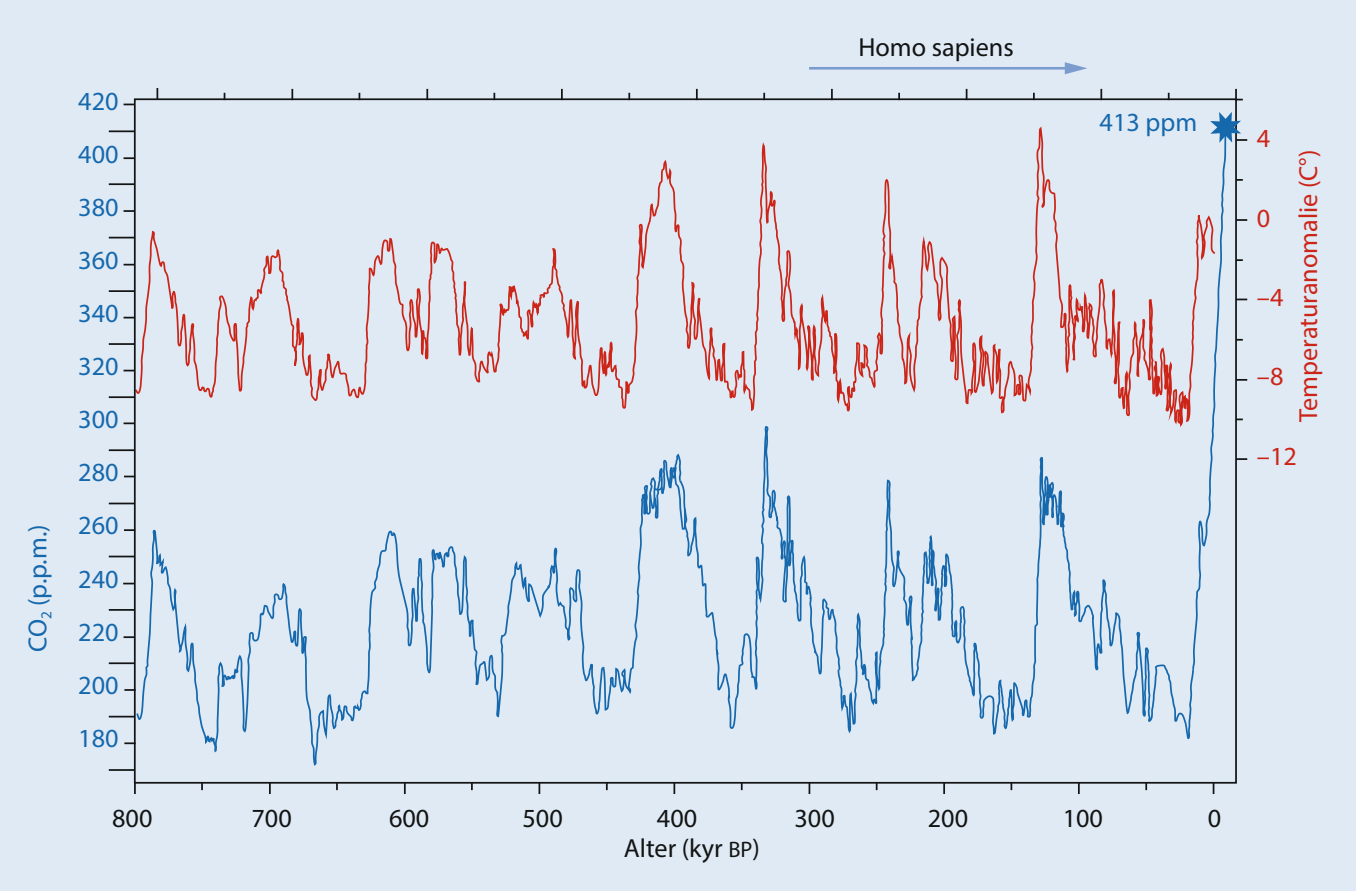

Abb. $1<$ Verlauf der Kohlendioxid $\left(\mathrm{CO}_{2}\right)$-Konzentration (ppm) und Temperatur, bezogen auf den Mittelwert $\left({ }^{\circ} \mathrm{C}\right)$ der letzten 800.000 Jahre, basierend auf Eisbohrkernuntersuchungen mehrerer Forschungsgruppen im Rahmen des „European Project for Ice Coring in Antarctica" (EPICA) [10]. Kyr Kilojahre (1000 Jahre), $B P$ "before present", blauer Stern aktuell gemessene $\mathrm{CO}_{2}$-Konzentration von 413 ppm in der Atmosphäre. (Modifiziert nach Luthi et al. [7])

Um diese Folgen abzumildern, sollten Anästhesisten ihren Teil dazu beitragen und den $\mathrm{CO}_{2}$-Fußabdruck des Gesundheitssystems reduzieren. Im Bereich der Anästhesiologie sind diesbezüglich folgende Themenbereiche von besonderer Relevanz: (I) Nutzung von inhalativen Anästhetika, (II) Energieversorgung der Krankenhäuser und (III) Abfallmanagement im OP-Bereich. Diese Aspekte werden im vorliegenden Beitrag ausführlich dargestellt. Bisher sind diese Zusammenhänge - Klimawandel und Anästhesieführung - nur wenigen Anästhesisten bekannt. Bei einer Umfrage in Australien und Neuseeland gaben nur $10 \%$ aller befragten Anästhesisten an, Umweltkriterien in die Wahl ihres Narkoseverfahrens einzubeziehen [13].

\section{Inhalative Anästhetika als Treibhausgase}

Alle volatilen Anästhetika sind halogenierte Kohlenwasserstoffe und damit hochpotente Treibhausgase. Isofluran und sein Enantiomer Enfluran enthalten ein Chloratom und sind damit Fluorchlorkohlenwasserstoffe (FCKW), genau wie Halothan, das zusätzlich ein Bromatom enthält. Durch ihr Chloratom haben FCKW-Substanzen außerdem eine ozondepletierende Wirkung. Sevofluran und Desfluran dagegen sind Fluorkohlenwasserstoffe (FKW).

Wie viel ein Treibhausgas zur Erderwärmung über einen bestimmten Zeitraum beiträgt, wird mithilfe der Global Warming Potenz (GWP) angegeben, wobei $\mathrm{CO}_{2}$ für diese Angaben als Bezugsgröße dient. Übliche Zeitspannen beziehen sich auf 20 oder 100 Jahre, dann als $\mathrm{GWP}_{20}$ und $\mathrm{GWP}_{100}$ bezeichnet. Die GWP eines Gases hängt von folgenden Faktoren ab [14-17]:

- atmosphärische Lebensdauer des Treibhausgases,

- Absorptionskraft über die gesamte atmosphärische Lebensdauer,

- Wellenlänge, auf der das Treibhausgas absorbiert. Es ist umso potenter, je weniger es in demselben Infrarotspektrum der natürlichen Treibhausgase Wasserdampf und $\mathrm{CO}_{2}$ absorbiert.

Die Einheit Kohlendioxidäquivalent $\left(\mathrm{CO}_{2 \text {-eq }}\right)$ wird genutzt, um die Emissionen verschiedener Treibhausgase, basierend auf ihrer GWP, zu vergleichen.

Aufgrund der immensen erderwärmenden Potenz von halogenierten Kohlenwasserstoffen wurde im Rahmen des Kyoto-Protokolls eine weltweite Reduk- tion vereinbart (2005), und 2016 wurde in Kigali ein weltweiter Verzicht bis 2035 unterzeichnet [18]. Ab Mitte des 21. Jh. haben halogenierte Kohlenwasserstoffe vermutlich einen substanziellen Einfluss auf die anthropogene Klimaerwärmung [19]. Volatile Anästheti$\mathrm{ka}$ wurden in beiden Abkommen ausgenommen, da es sich um medizinisch notwendige Substanzen handelt. Somit sind sie heute die einzigen halogenierten Kohlenwasserstoffe, deren Konzentrationen in der Atmosphäre weiter ansteigen [20]. Der Verbrauch an volatilen Anästhetika ist in den letzten Jahrzehnten steil gestiegen und wird weitersteigen, einerseits durch eine verbesserte medizinische Versorgung in den aufstrebenden Entwicklungsländern und andererseits durch die wachsende Alterung in den westlichen, hochentwickelten Staaten, da ältere Patienten im Durchschnitt häufiger eine Operation benötigen.

In Deutschland wurden 2012 bei ca. 7 Mio. Operationen inhalative Anästhetika eingesetzt, wobei die Anteile von Sevofluran ca. $55 \%$, von Desfluran ca. $35 \%$ und von Isofluran ca. $10 \%$ betrugen [21]. Weltweit haben volatile Anästhetika 2014 ein $\mathrm{CO}_{2 \text {-eq }}$ von 3 Mio. Tonnen verursacht, $80 \%$ davon allein durch Desfluran [22]. Desfluran hat von 
allen volatilen Anästhetika mit Abstand den größten Einfluss auf die Erderwärmung [15], was einerseits durch die längere atmosphärische Lebensdauer und andererseits durch die höhere radiative Effektivität bedingt ist ( $\bullet$ Tab. 1; [15, 16]). Das $\mathrm{GWP}_{20}$ von Desfluran beträgt 6810, was bedeutet, dass sein Treibhauseffekt 6810-mal ausgeprägter ist als der von $\mathrm{CO}_{2}$. Das $\mathrm{GWP}_{100}$ von Desfluran beträgt noch $2540[14,15]$. Sevofluran hat ein $\mathrm{GWP}_{20}$ von 440 , für Isofluran beträgt das $\mathrm{GWP}_{20}$ 1800. Lachgas übt aufgrund seiner extrem langen atmosphärische Lebensdauer (114 Jahre) und seiner zusätzlichen ozondepletierenden Wirkung nach Desfluran den zweitgrößten Klimaeffekt über den Zeithorizont von 100 Jahren aus [14]. Das $\mathrm{GWP}_{100}$ von Lachgas beträgt 300 , wobei ca. $1 \%$ der gesamten atmosphärischen Lachgaskonzentration durch die medizinische Nutzung verursacht wird.

Bei inhalativen Anästhetika sollten für eine adäquate Einschätzung des GWP und des $\mathrm{CO}_{2 \text {-eq }}$ die unterschiedlichen klinisch angewandten Konzentrationen und die Frischgasflüsse (FGF) mit einbezogen werden $[14,23]$. Da für eine Allgemeinanästhesie mit Desfluran im Vergleich zu Isofluran und Sevofluran deutlich höhere Gaskonzentrationen notwendig sind, findet sich ein noch ausgeprägterer Unterschied bei Betrachtung des GWP und des $\mathrm{CO}_{2 \text {-eq }}[14,23$, 24].

Volatile Anästhetika üben ihren maximal schädlichen Effekt innerhalb ihrer atmosphärischen Lebensdauer aus. Das Konzept der $\mathrm{GWP}_{20}$ und $\mathrm{GWP}_{100}$ zeigt bei solch relativ kurzlebigen Substanzen nur einen Residualeffekt auf, der besteht, wenn die eigentliche Substanz bereits eliminiert ist, und unterschätzt somit die schädigende Potenz in den ersten Jahren nach der Gasemission [24]. Özelsel et al. haben die erderwärmende Potenz auch für den Zeithorizont von einem Jahr berechnet (GWP ; - Tab. 2). Eine 7 -stündige Narkose bei einem Frischgasfluss von $0,5 \mathrm{l} / \mathrm{min}$ mit $2 \%$ Sevofluran verursacht einen Treibhausgaseffekt, vergleichbar mit einer $783 \mathrm{~km}$ langen Autofahrt; bei $1,2 \%$ Isofluran sind es $667 \mathrm{~km}$ und bei $6 \%$ Desfluran $3924 \mathrm{~km}$. In Abhängigkeit vom FGF steigen diese

Anaesthesist 2020 - 69:453-462 https://doi.org/10.1007/s00101-020-00770-1

(c) Der/die Autor(en) 2020

\section{S. Koch.S. Pecher}

\section{Neue Herausforderungen für die Anästhesie durch den Klimawandel}

\section{Zusammenfassung}

Hintergrund. Die Klimakrise stellt die größte Bedrohung der globalen Gesundheit im 21. Jh. dar. Treibhausgasemissionen westlicher Länder sind zu 5-10\% dem Gesundheitssektor zuzurechnen; hierzu tragen energieintensive Abteilungen (Intensivmedizin, OP-Bereich und Rettungsdienst) maßgeblich bei. Ziel der Arbeit. Ziel der Arbeit ist es, Hintergrundwissen und praktische Tipps zum Erreichen eines klimafreundlichen Krankenhauses zu geben.

Material und Methoden. Narrativer Review mit Hintergrundwissen zu den Themenfeldern: (I) inhalative Anästhetika als Treibhausgase, (II) Energieversorgung im Krankenhaus und (III) Abfallwirtschaft. Ergebnisse und Diskussion. (I) Volatile Anästhetika sind hochpotente Treibhausgase; vor allem Desfluran sticht mit einer sehr hohen Global Warming Potenz (GWP) heraus. Einen geringeren Klimaeinfluss haben die totale intravenöse Anästhesie (TIVA) oder Regionalanästhesieverfahren. (II) Krankenhäuser sollten auf erneuerbare Energien umsteigen und gezielte Energiesparmaßnahmen (Leuchtdioden [LED], Bewegungssensoren etc.) implementieren. (III) Ein Abfallmanagement nach dem Konzept „Reduce, reuse, recycle, rethink, research" kann die Müllproduktion im Krankenhaus reduzieren. Ärzte/Ärztinnen sollten aktiv dazu beitragen, die Klimaziele zu erreichen.

\section{Schlüsselwörter}

Treibhausgase · Globale Erwärmung · Inhalationsanästhetika · Erneuerbare Energie . Abfallmanagement

\section{New challenges for anesthesia due to the climate change}

\section{Abstract}

Background. The climate crisis is the most serious threat to global health in the twentyfirst century. In western countries $5-10 \%$ of all greenhouse gas emissions originate from the healthcare sector and the main contributing factors are energy-intense departments (intensive care units, operating suits and prehospital emergency services).

Objective. The aim of this review is to provide background knowledge and practical ideas to achieve climate-neutral hospitals.

Material and methods. Narrative review with information on the topics of (I) volatile anesthetics as greenhouse gases, (II) energy supply in hospitals and (III) solid waste management.

Results and conclusion. (I) Volatile anesthetics are highly potent greenhouse gases, especially desflurane has a major global warming potential. Total intravenous anesthesia (TIVA) with propofol or regional anesthetic techniques have a much lower impact on the climate. (II) Using sustainable energy sources as well as initiating energy sparing techniques, such as light-emitting diodes (LED) and motion sensors, can reduce $\mathrm{CO}_{2}$ emissions. (III) Waste can be managed by the reduce, reuse, recycle, rethink and research concept. Doctors should actively contribute to reach the climate goals.

\section{Keywords}

Greenhouse gases - Global warming · Inhalation anesthetics - Renewable energy . Waste management
Zahlen deutlich an. Beachte: Bei einer Anästhesie mit 6\%igem Desfluran und einem FGF von 21 entspräche dies einer Fahrt vom Nordkap in Norwegen bis nach Kapstadt in Südafrika oder $15.698 \mathrm{~km}$ in nur $7 \mathrm{~h}$. Dies verdeutlicht die Wichtigkeit von "Low-flow“Techniken in der Anästhesie, um Treibhausgasemissionen zu vermindern [23, 24]. Online stehen 2 kostenlose Apps zur
Verfügung, die den $\mathrm{CO}_{2 \text {-eq }}$ berechnen (allerdings basierend auf dem Zeithorizont von 100 Jahren), indem man den FGF und die Konzentration des Anästhetikums eingibt (Anaesthetic Impact Calculator und Yale Gassing Greener).

Im Gegensatz zur Nutzung inhalativer Anästhetika hat eine intravenöse Anästhesie mit Propofol einen deutlich geringeren Einfluss aufdie Erderwärmung, wie 
Tab. 1 Klinische und atmosphärische Charakteristika inhalativer Anästhetika

\begin{tabular}{|c|c|c|c|c|}
\hline & Isofluran & $\begin{array}{l}\text { Sevo- } \\
\text { fluran }\end{array}$ & Desfluran & Lachgas \\
\hline Chemische Formel & $\mathrm{C}_{3} \mathrm{H}_{2} \mathrm{ClF}_{5} \mathrm{O}$ & $\mathrm{C}_{4} \mathrm{H}_{3} \mathrm{~F}_{7} \mathrm{O}$ & $\mathrm{C}_{3} \mathrm{H}_{2} \mathrm{~F}_{6} \mathrm{O}$ & $\mathrm{N}_{2} \mathrm{O}$ \\
\hline Atmosphärische Konzentration 2014 (ppt) & 0,12 & 0,23 & 0,32 & $328(\mathrm{ppb})$ \\
\hline Atmosphärische Lebenspanne (Jahre) ${ }^{\mathrm{a}}$ & 3,2 & 1,1 & 14 & 114 \\
\hline Radiative Effizienz $\left(\mathrm{W} \mathrm{m}^{-2} \mathrm{ppb}^{-1}\right)^{\mathrm{a}}$ & 0,453 & 0,351 & 0,469 & $4,210^{-6}$ \\
\hline$G W P_{20}{ }^{a}$ & $1800^{\mathrm{a}}$ & $400^{\mathrm{a}}$ & $6810^{\mathrm{a}}$ & $290^{\mathrm{b}}$ \\
\hline$G W P_{100}{ }^{a}$ & $510^{\mathrm{a}}$ & $130^{\mathrm{a}}$ & $2540^{\mathrm{a}}$ & $300^{\mathrm{b}}$ \\
\hline $\begin{array}{l}C D E_{20} \text { (FGF 21/min, } M A C_{50} \text { in Luft) } \\
\text { (Isofluran 1,25\%; Sevofluran 2\%; Desfluran 6\%) }\end{array}$ & 15.551 & 6980 & 187.186 & - \\
\hline $\begin{array}{l}C D E_{20} \text { (FGF 2l/min, } M A C_{50} \text { mit Lachgas) }{ }^{b} \\
\text { (Isofluran 0,5\%; Sevofluran 0,8\%; Desfluran } \\
2,4 \% \text { ) }\end{array}$ & 44.610 & 40.940 & 113.022 & - \\
\hline $\begin{array}{l}C D E_{20}\left(F G F 0,51 / \text { min, } M A C_{50} \text { in Luft }\right)^{b} \\
\text { (Isofluran 1,25\%; Sevofluran 2\%; Desfluran 6\%) }\end{array}$ & 3881 & - & 46.796 & - \\
\hline \multicolumn{5}{|c|}{ 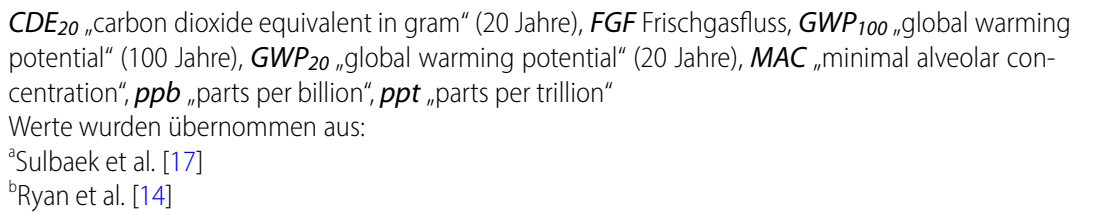 } \\
\hline
\end{tabular}

Tab. 2 Kilometeräquivalentinhalativer Anästhetika mitunterschiedlichen Frischgasflüssenzum $\mathrm{GWP}_{1}$ für $7 \mathrm{~h}$ Narkose. (Werte wurden übernommen aus Ozelsel et al. [24])

\begin{tabular}{|c|c|c|c|c|}
\hline \multirow{2}{*}{$\begin{array}{l}\text { Frischgasfluss } \\
\text { (I/min) }\end{array}$} & \multicolumn{4}{|c|}{ Kilometeräquivalent (km) } \\
\hline & $\begin{array}{l}\text { Isofluran } \\
(1,2 \%)\end{array}$ & $\begin{array}{l}\text { Sevofluran } \\
\text { (2\%) }\end{array}$ & $\begin{array}{l}\text { Desfluran } \\
(6 \%)\end{array}$ & $\begin{array}{l}\text { Lachgas } \\
(66 \%)\end{array}$ \\
\hline 0,5 & 667 & 783 & 3924 & 279 \\
\hline 1 & 1334 & 1566 & 7849 & 558 \\
\hline 2 & 2668 & 3132 & 15.698 & 1116 \\
\hline
\end{tabular}

in einer Life Cycle Analyse (LCA) unter Einbeziehung von Herstellung, Verpackung, Transport, Applikation und Abfallmanagement für inhalative Anästhetika und Propofol gezeigt werden konnte [23]. Auch Regionalanästhesieverfahren haben einen geringeren Einfluss auf die globale Erderwärmung, da weniger Material und weniger inhalative Anästhetika verbraucht werden.

Primär sollten Sevofluran oder Isofluran genutzt werden, während Desfluran und Lachgas nur zur Anwendung kommen sollten, wenn dies klinisch indiziert ist (ggf. Wachkraniotomien für Desfluran, [23, 24]). Valide Alternativen zur Gabe von Lachgas, v.a. im geburtshilflichen Bereich, sollten erforscht werden [25]. Es sollten zeitnah Technologien zur Marktreife gebracht werden, die volatile Anästhetika absorbieren und wieder aufbereiten [23, 26]. Dies ist keine uner- reichbare Zukunftsmusik, sondern es gibt schon mittelständige Firmen, die über die entsprechende Technologie verfügen [27]. Allerdings bedarf es dringend staatlicher Förderung, um diese Systeme zeitnah in ausreichender Menge zu produzieren und in möglichst vielen Kliniken installieren zu können. Auch die Pharmaindustrie sollte entsprechende Forschung vorantreiben, um die Entwicklung von Anästhesiegasen ohne Einfluss auf globale Erwärmung voranzutreiben.

\section{Energieversorgung im Krankenhaus}

Krankenhäuser sind 24h/Tag und 7 Tage/ Woche in Betrieb und sind energieintensive Verbraucher. In einer Studie von MacNeill et al. wurde der $\mathrm{CO}_{2-}$ Fußabdruck über den Zeitraum von einem Jahr (2011) an 3 großen Klini- ken in USA (University of Minnesota Medical Center), Großbritannien (John Radcliffe Hospital) und Kanada (Vancouver General Hospital) verglichen. Insgesamt lagen die $\mathrm{CO}_{2 \text {-eq-Emissionen }}$ für alle drei Kliniken in einem Bereich von 3220-5190t $\mathrm{CO}_{2 \text {-eq }} / \mathrm{Jahr}$, wobei die Emissionen vor allem dem Energiesektor (Elektrizität, Heizung und Klimaanlage) sowie der Nutzung von inhalativen Anästhetika zuzurechnen waren [28]. Während in Großbritannien und den USA hauptsächlich mit Kohle geheizt wurde, erhielt das Vancouver General Hospital seine Energie vornehmlich von Wasserkraftwerken und reduzierte damit seinen $\mathrm{CO}_{2}$-Fußabdruck um den Faktor 10. Dieses Beispiel macht deutlich, dass Krankenhäuser v.a. mit einem Umstieg auf erneuerbare Energien in eine lebenswerte, gesunde Zukunft investieren können (•Tab. 3). Gerade bei öffentlichen Gebäuden, wie Krankenhäusern, Schulen und Behörden, ist die Installation von Photovoltaikanlagen sehr wirtschaftlich, da ein Großteil der Energienutzung stattfindet, während die Sonne am Himmel steht - im Gegensatz zu Eigenheimen oder Mietshäusern. Die Energieeffizienz kann durch Optimierung der Gebäudesubsatz deutlich verbessert werden [29].

Kraft-Wärme-Kopplungsanlagen (KWK - auch Blockheizkraftwerk), die in einigen deutschen Krankenhäusern betrieben werden, haben eine hohe Energieausbeute. Die KWK-Anlagen nutzen die bei der Stromerzeugung anfallende Wärme zum Heizen. Diese hohe Energieausbeute ist jedoch nur bei optimalem Betrieb der KWK-Anlagen möglich, wenn z.B. im Winter die Abwärme der Stromerzeugung auch wirklich zum Heizen benötigt wird [30]. Insgesamt sind die $\mathrm{CO}_{2}$-Einsparungen von KWK-Anlagen zu gering, um einen wirksamen Klimaschutz voranzutreiben, solange sie mit fossilen Energieträgern betrieben werden. Werden KWK-Anlagen auf den Betrieb mit regenerativen Energieträgern umgerüstet (z.B. Biomasse, regenerativ erzeugter Wasserstoff, regenerativ erzeugtes Methan oder Geothermie), können sie Strom und Wärme bei gleichzeitig guter Energieausbeute 
Tab. 3 Anteil der $\mathrm{CO}_{2}$-Emissionen von Krankenhäusern und ihr Einsparpotenzial. (Basierend auf Daten von MacNeill et al. [28])

\section{Anteilige Spannbreite der Krankenhaus- $\mathrm{CO}_{2}$ -} Emissionen (\%)

\begin{tabular}{|c|c|c|}
\hline Anästhetikanutzung & $4-63$ & $\begin{array}{l}\text { Keine Nutzung von Desfluran } \\
\text { reduziert die } \mathrm{CO}_{2} \text {-Emissionvon } \\
63 \% \text { auf } 4 \%\end{array}$ \\
\hline \multirow[t]{2}{*}{$\begin{array}{l}\text { Energiesektor (Heizung, } \\
\text { Klimaanlage, Ventilation, } \\
\text { Strom) }\end{array}$} & \multirow[t]{2}{*}{$17-84$} & $\begin{array}{l}\text { Nutzung von erneuerba- } \\
\text { ren Energien reduziert die } \\
\mathrm{CO}_{2} \text {-Emission von } 84 \% \text { auf } \\
17 \%\end{array}$ \\
\hline & & $\begin{array}{l}\text { Umsetzung von Energiespar- } \\
\text { maßnahmen reduziert die } \\
\mathrm{CO}_{2} \text {-Emission von } 84 \% \text { auf } \\
36 \%\end{array}$ \\
\hline $\begin{array}{l}\text { Abfallmanagement (Hausmüll, } \\
\text { toxischer/infektiöser Abfall, } \\
\text { Wasserverbrauch, wiederver- } \\
\text { wendbare Textilien ...) }\end{array}$ & $12-20$ & $\begin{array}{l}\text { Keine großen Differenzen bei } \\
\text { den } 3 \text { untersuchten Kliniken }\end{array}$ \\
\hline $\begin{array}{l}\text { Es zeigt sich, dass im Bereich der } \\
\text { Energien, die „Nichtnutzung" von } \\
\text { großen Einfluss haben können }\end{array}$ & $\begin{array}{l}\text { anästhes } \\
\text { Desflurz }\end{array}$ & $\begin{array}{l}\text { e Umstellung auf erneuerbare } \\
\text { g von Energiesparmaßnahmen }\end{array}$ \\
\hline
\end{tabular}

komplett $\mathrm{CO}_{2}$-neutral erzeugen - das Ziel der Zukunft [30].

Weiterhin kann durch gezielte Ma $\beta$ nahmen wie die Umrüstung auf LEDLeuchtmittel, das Absenken bzw. Ausstellen von Klimaanlagen im OP nachts und am Wochenende (bis auf die Not-OP-Säle), Bewegungssensoren in nachts wenig genutzten Gängen und Treppenhäusern, gut gewarteten Geräten wie Kühlschränke, Sterilisatoren und Heizungen der Energiebedarf deutlich gesenkt werden [28]. Die effiziente Umsetzung von Energiesparmaßnahmen kann die $\mathrm{CO}_{2}$ Emissionen von Krankenhäusern um 30-50\% reduzieren [28].

Das Projekt „KLIK green“, eine Initiative des Bund für Umwelt und $\mathrm{Na}$ turschutz Deutschland (BUND) und des Bundesumweltministeriums, bietet 250 interessierten Kliniken an, einen Klinikmitarbeiter zu einem Klimamanager auszubilden, um die oben genannten Einsparungen mithilfe der erworbenen Expertise möglich zu machen [31].

\section{Abfallmanagement im Operationssaal}

20-30\% des Krankenhausmülls entstehen im OP, davon ca. $25 \%$ durch die Anästhesie [32]. Bei einem wachsenden Anteil an Einmalprodukten und aufgrund
Sets/Überschuss. Vorgefertigte Sets für die Anlage von Kathetern oder Regionalanästhesie erleichtern die Arbeit, enthalten aber häufig unnötige Materialien wie Kompressen, Lochtücher, Instrumente oder Plastikschalen. Als „Überschuss" wird das Öffnen von sterilem Equipment bezeichnet, das schlussendlich nicht genutzt wird, aber aus hygienischen Gründen verworfen werden muss. Bis zu $20 \%$ des Materials einer OP bleiben ungenutzt und müssen verworfen werden [36]. Hersteller können mit wenig Aufwand solche Sets neu packen und nur mit relevanten Utensilien bestücken, was ökologisch $\mathrm{zu}$ fordern und gleichzeitig kosteneffektiv ist.

Medikamente. Entsprechend werden auch Medikamente, aufgezogen für den Notfall, in mehr als $50 \%$ der Fälle verworfen [37]. Bei „schwierig“ aufzuziehenden Medikamenten oder Verdünnungen (z.B. Katecholamine) oder bei Medikamenten, die schnell verfügbar sein müssen (z. B. Notsectio), empfiehlt sich die Erwägung von in der Apotheke vorgefertigten Spritzen, die länger haltbar sind und somit den Verwurf reduzieren. Generell sollte die Ampullengröße dem Verbrauch entsprechen. Durch eine Umstellung von Propofol von 50-ml- bzw. 100-ml-Amp. auf 20-ml-Amp. konnte beispielsweise der Verwurf um ca. 90\% reduziert werden [38].

Sonstiges. Kaffeebecher, Kaffeekapseln, Plastikflaschen und sonstige Einwegartikel werden im privaten Haushalt bereits oft vermieden; dies sollte auch am Arbeitsplatz selbstverständlich sein. Wiederfüllbare Druckerpatronen und Akkus statt Batterien sind empfehlenswert. Der Papierverbrauch kann durch doppelseitiges Ausdrucken sowie die Nutzung elektronischer Patientenakten und Anästhesiedokumentation reduziert werden, wenn diese konsequent zum Einsatz kommen. Allerdings haben auch die elektronischen Alternativen einen negativen Umwelteinfluss, solange die Umstellung auf erneuerbare Energien nicht 100\%ig umgesetzt ist. Verwendung von Recyclingpapier sollte Standard sein. 
Tab. 4 Ideen-Checkliste für den Beginn eines ökologischen Konzepts in einer anästhesiologischen Abteilung

\begin{tabular}{l} 
Themenbereiche \\
\hline Kooperation aller
\end{tabular}

Mitarbeiter

Anästhetika

Energiemanagement

Energien umstellen

Gut gewartete und energiesparende elektrische Geräte einsetzen

Klimaanlagen im OP nur zu Betriebszeiten einschalten

Bewegungssensoren in Treppenhäusern/Fluren anbringen

Flächendeckend LED-Lampen nutzen

Abfallmanagement
1) Reduce

2) Reuse

3) Recycle

4) Rethink

5) Research

Mobilität

Beatmungsschläuche 7 Tage verwenden

Restriktiv bestückte Fertigsets benutzen

Überschuss vermeiden

Anwendung von Fertigspritzen erwägen

Adäquate Ampullengrößen wählen

Verantwortungsbewussten Papierdruck betreiben

Einsatz von Mehrwegprodukten erwägen

OP-Textilien

Larynxmasken

Laryngoskope

Medikamentenschalen

Konzepte für den OP entwickeln

Papier/Karton

Plastik

Glas

Metall

Nachhaltigkeitskonzepte der Industriepartner prüfen

Gesunde Küche

"Divestment"

Audits etablieren

Benchmarking

Ökologische Forschungsprojekte durchführen

Berufswege

Öffentliche Verkehrsmittel nutzen/Job-Tickets anbieten

Bau von Radwegen/Radstellplätzen fördern

Bildungsreisen

Lokale Fortbildungen besuchen

Flugreisen vermeiden

Telekonferenzen/Kongress-Streaming anbieten

Details s. Text. Je nach örtlichen Gegebenheiten ergeben sich viele weitere Möglichkeiten zur ökologischen Nachhaltigkeit

LED "light-emitting diode", TIVA totale intravenöse Anästhesie

\section{Reuse}

Einmalinstrumente und Equipment sind überall präsent und werden stetig mehr. Bei der Entscheidung zwischen Einmal- und Mehrwegprodukten kommen sowohl hygienische Bedenken, Kostenfaktoren und als auch Bequemlichkeit zum Tragen. Häufig eingesetzte Einwegartikel sind Textilien (OP-Mäntel und -Abdecktücher), Medikamentenschalen, Beatmungsschläuche, Larynxmasken, aber auch Laryngoskopspatel und -handgriffe, Ambubeutel, Pulsoxymetersonden, Führungsstäbe und sogar Blutdruckmanschetten finden zunehmend Anwendung. Um den Umwelteinfluss und den $\mathrm{CO}_{2}$-Fußabdruck eines Einmalartikels mit dem eines Wiederverwertbaren $\mathrm{zu}$ vergleichen, werden LCA angewandt. In die Untersuchung wird einbezogen, wo und wie Rohstoffe gewonnen werden, deren Verarbeitung und die Herstellung des Produkts, Transportwege, Gebrauch, Wiederverwertung und Instandhaltung, Recycling und $\mathrm{Ab}$ fallbeseitigung [39].

Beider Entscheidung zwischen waschbaren und Einmal-OP-Mänteln und -Abdecktüchern spielen v.a. Schutz vor Infektion von Personal und Patient, Tragekomfort sowie ökonomische und ökologische Aspekte eine Rolle [40]. Unterschiede bezüglich der Inzidenz von Wundinfektionen zwischen modernen OP-Textilien mit Einfach- oder Mehrfachgebrauch sind nicht nachweisbar; beide Optionen erfüllen die notwendigen Standards. Aus ökologischer Sicht ist die Datenlage eindeutig: Alle 6 verfügbaren LCA ergaben eine klare Überlegenheit der wiederverwertbaren Textilien. Diese sparen $200-300 \%$ an Energie, reduzieren den Wasserverbrauch um 250-330\% und verringern die Müllproduktion um $750 \%$ gegenüber den Einmalprodukten [40].

Wiederverwertbare Larynxmasken haben bei einer LCA in den Kategorien GWP, Wasserverbrauch und Luftverschmutzung insgesamt einen ca. 50\% geringeren negativen ökologischen Effekt gegenüber der entsprechend notwendigen Zahl von Einmallarynxmasken ergeben [41]. 
Bei der Nutzung von Einmallaryngoskopen sind die $\mathrm{CO}_{2}$-Emission 16- bis 25-mal höher als bei Gebrauch der herkömmlichen Laryngoskope aus rostfreiem Stahl, einschließlich einer „High-level“-Desinfektion. Bezüglich der Spatel war der Unterschied 6- bis 8-fach zugunsten der wiederverwertbaren Optionen [42]. Wie bei vielen medizinischen Produkten werden insbesondere bezüglich der Instrumente zur Atemwegssicherung hygienische Bedenken als Hauptargument für Einmalprodukte angeführt. Einwegmaterialien sind sicherlich wertvoll, wenn adäquate Dekontaminationsmöglichkeiten fehlen. Ein unselektiver Einsatz von Einmalprodukten sollte jedoch vermieden werden, da diese den ökologischen Fußabdruck des Gesundheitssystems durch den vermehrten Verbrauch an Ressourcen bei der Produktion, dem Transport und dem Abfallmanagement erhöhen, ohne einen messbaren Effekt auf das Infektionsrisiko zu demonstrieren [43].

Mehrweg-Medikamentenschalen zeigen eine deutliche Reduktion an Wasserverbrauch und Kosten im Vergleich zu Einmalprodukten, die $\mathrm{CO}_{2}$-Produktion reduzierte sich aber nur geringfügig. Dies ist v. a. dadurch zu erklären, dass diese LCA in Victoria, Australien, durchgeführt wurde, wo Braunkohle als Hauptenergiequelle dient. In einem Krankenhaus, das mit erneuerbaren Energieträgern arbeitet, ergäbe sich eine $\mathrm{CO}_{2}$ - $\mathrm{Re}$ duktion von $>50 \%$ [44].

Im Gegensatz dazu zeigte sich für zentrale Venenkatheter (ZVK), dass bei der Nutzung von Mehrwegsets aufgrund des Sterilisierungsvorgangs mehr Wasser und $\mathrm{CO}_{2}$ verbraucht wurden als beim Gebrauch von Einmalsets [45].

\section{Recycle}

Müll aus dem OP besteht aus den unterschiedlichsten Materialien: Plastik, Papier, Karton, Glas, Metall, Anästhesiegase, Medikamente, Spitzabfall und anatomisch-pathologischer Abfall. Etwa 60\% des Krankenhausmülls sind nicht kontaminiert und somit potenziell recycelbar. Besonders viel dieses „sauberen“ Mülls fällt am Anfang einer Operation an, wenn die Tische gerichtet und Verpackungen geöffnet werden [46]. Weitere $30 \%$ sind als potenziell kontaminiert zu betrachten und können somit nicht recycelt werden. Nur ca. $10 \%$ sind infektiös oder enthalten Schadstoffe wie Chemikalien oder Zytostatika. Diese müssen getrennt gesammelt und in speziellen Sonderabfallverbrennungsanlagen unter hohen $\mathrm{CO}_{2}$ Emission entsorgt werden [47].

Eine Umfrage unter 780 Anästhesisten aus England, Australien und Neuseeland ergab, dass $>90 \%$ der Anästhesisten im privaten Haushalt recyceln, $>90 \%$ auch im Krankenhaus recyceln möchten, dies aber nur ca. $11 \%$ am Arbeitsplatz durchführen. Als größtes Hindernis zum effektiven Recycling im OP wurden in allen Ländern fehlende Recycling-Tonnen angegeben; des Weiteren wurden die Haltung der Mitarbeiter zum Thema und mangelnde Informationen genannt [48].

Lokale Recyclingfirmen sollten zu Rate gezogen werden, was, wo und wie am besten gesammelt und abtransportiert werden kann.

\section{Rethink}

Neue Ideen sind gesucht - statt „business as usual“ [49]. Wie können der Einleitungsraum und der OP so gestaltet werden, dass Recycling einfach wird? Pharma- und Produktfirmen sollten auf ihre Umweltfreundlichkeit bei der Herstellung ihrer Waren geprüft werden.

Interdisziplinäre „Green Teams“, die sich mit dem Thema Ökologie im OP beschäftigen, können viel erreichen. $\mathrm{Ne}$ ben Ideensammlung und deren zeitnaher Umsetzung sollte eine Schulung des Personals erfolgen, um allgemein eine umweltfreundliche Atmosphäre zu schaffen. Voraussetzung für ein effizientes Arbeiten ist, dass sich diese Teams aus Personen in Schlüsselpositionen wichtiger Bereiche zusammensetzen: OP-Management, Ärzte und Pflegepersonal, Hygiene, Techniker, Abfallbeauftragte, Pharmazeuten und Verwaltung. Strategien für Kliniken und ihre „Green Teams" sind bereits verbreitet und weltweit etabliert [50].

Das Zurücklegen des Arbeitswegs mithilfe des öffentlichen Nahverkehrs, des Fahrrads oder gar zu Fuß sollte unbedingt gefördert werden, z. B. mit der Etablierung günstiger Job-Tickets. Rei- sen zu Konferenzen sollten mit der Bahn statt mit dem Flugzeug für alle Strecken unter $800 \mathrm{~km}$ gefordert werden; auch Telekonferenzen sind heutzutage leicht umsetzbar.

Regionale, biologisch angebaute Obstund Gemüseprodukte, vegetarische oder sogar vegane Mahlzeiten sollten im Sinne einer gesunden Ernährung in der Patientenverpflegung und in der Personalkantine häufiger angeboten werden.

Divestment bezeichnet das Abziehen aller Kapitalanlagen von Industrien, die zur Klimakrise beitragen. In der Finanzwelt gelten Geldanlagen in klimaschädlichen Bereichen als größtes Investmentrisiko [51-53]. In einem aktuellen Editorial des British Medical Journal (BMJ) rufen die Autoren sowohl alle Mitarbeiter des Gesundheitssystems als auch alle medizinischen Organisationen dazu auf, Divestment als Werkzeug zu nutzen, um Politik und Industrie zu einem der Gesundheit des Planeten und der Menschheit förderlichen Verhalten zu bewegen [54]. Die Berliner Ärzteversorgung hat schon 2006 begonnen, ihr Aktienfondsvermögen auf nachhaltige Kapitalanalgen umzuschichten, und wurde dafür 2017 von der Organisation Fossil Free Berlin mit dem „Berlin Divestment Award“ ausgezeichnet [55]. Als weitere lobenswerte Beispiele aus dem medizinischen Bereich sind das Royal College of Physicians, das Royal College of General Practitioners, das Royal Australasian College of Physicians, die Medical Associations von America and Kanada sowie die Britisch Medical Association zu nennen [56].

\section{Research}

Am Anfang jeder Änderung sollte eine Bestandsaufnahme stehen. Ein Audit zu verschiedenen Themen kann Aufschluss über die Effektivität der eigenen Praxis geben und den Effekt einer etwaigen Veränderung verfolgen. Im Sinne eines Benchmarkings kann sich die eigene Abteilung oder Klinik an anderen vergleichbaren Einrichtungen messen. Ein Beispiel hierfür ist das Projekt: „Inhaled Anesthesia Climate Initiative: Project Drawdown“.

Audits sind ebenfalls sinnvoll, um beispielsweise den Verbrauch von Lach- 


\section{Infobox 1 Resolution des Weltärztebundes „World Medical Association" vom 18.11.2019a \\ Die "World Medical Association" und ihre Gründungsmitglieder sowie internationale Gesundheitsorganisationen ... \\ - rufen den Klimanotstand aus und fordern die internationale Gemeinschaft der Gesundheitsberufe auf, sich dieser Mobilisierung anzuschließen \\ - verpflichten sich dafür einzutreten, die Gesundheit der Menschen weltweit vor den Klimafolgen zu schützen \\ - fordern von nationalen Regierungen, bis 2030 die $\mathrm{CO}_{2}$-Neutralität zu erreichen, um die lebensbedrohlichen Gesundheitsfol- gen der Klimakrise zu minimieren \\ - erkennen den ökologischen Fußabdruck des globalen Gesundheitssektors an, werden aktiv Müll reduzieren und $\mathrm{CO}_{2}$-Emissionen vermeiden, um ein nachhaltiges Gesundheitssystem zu gewährleisten}

aÜbernommen aus [57], frei übersetzt von den Autorinnen des vorliegenden Beitrags.

gas $\mathrm{zu}$ messen, und um festzustellen, wie oft TIVA oder Regionalanästhesien durchgeführt werden oder wie häufig die Low-flow-Anästhesie tatsächlich genutzt wird. Auch Themen wie Müllproduktion, Energieverbrauch und Gebrauch von Einmalmaterialien stellen gute AuditProjekte dar. Forschungsprojekte zum Thema Umweltschutz und klimafreundliche Anästhesie sollten gefordert und gefördert werden. Beispielsweise sind LCA aus Deutschland wichtig, ebenso wie die zeitnahe Entwicklung alternativer, klimafreundlicher Anästhesiegase oder von Anästhesiegaswiederaufbereitungsverfahren.

Innerhalb der weltweiten Gesundheitsorganisationen herrscht zunehmend Einigkeit, dass Aktionen, die die Klimakrise abmildern können, deutlich beschleunigt werden müssen. Der Weltärztebund hat im Oktober 2019 den Klimanotstand ausgerufen und bis 2030 die Klimaneutralität eingefordert (- Infobox 1).

\section{Fazit für die Praxis}

Der Glaube an ein ungebremstes Wachstum hat unseren Planeten Erde an seine Grenzen gebracht. Ein Über- leben der Menschheit auf der Erde ist nur möglich, wenn wir jetzt, hier und heute einen Wandel vollziehen und das Wohlergehen und die Gesundheit der Menschen und unserer Umwelt zum obersten Ziel erklären. Dieser Systemwandel ist kein Verzicht, sondern führt zu einer besseren Gesundheit, einem Gewinn an Lebensqualität und Lebenslust. Wir fordern alle Anästhesisten und Anästhesistinnen auf:

- auf Desfluran und Lachgas möglichst zu verzichten

- Sevofluran nur als low-flow Narkose zu nutzen

- TIVA und Regionalanästhesien bevorzugt anzuwenden.

- Klinikinterne „Green Teams“ zu bilden, um Energiesparmaßnahmen aufzudecken und umzusetzen.

- Müllvermeidung beginnt mit „reduce, reuse, recycle, rethink, research"

- Krankenhäuser sollten auf erneuerbare Energien umstellen und Photovoltaikanlagen installieren

Der Weltärztebund hat im Oktober 2019 den Klimanotstand ausgerufen und Klimaneutralität bis 2030 gefordert (- Infobox 1) [57]. Es ist Zeit zu handeln.

\section{Korrespondenzadresse}

\section{Dr. S. Koch}

Klinik für Anästhesiologie mit Schwerpunkt operative Intensivmedizin, Charité Universitätsmedizin Berlin, Campus VirchowKlinikum und Campus Mitte

Augustenburger Platz 1, 13353 Berlin, Deutschland

susanne.koch@charite.de

Danksagung. Die Autorinnen bedanken sich bei Frau Mone Schlegel für die Bearbeitung der - Abb. 1.

\section{Einhaltung ethischer Richtlinien}

Interessenkonflikt. S. Koch und S. Pecher geben an, dass kein Interessenkonflikt besteht.

Für diesen Beitrag wurden von den Autoren keine Studien an Menschen oder Tieren durchgeführt. Für die aufgeführten Studien gelten die jeweils dort angegebenen ethischen Richtlinien.

Open Access. Dieser Artikel wird unter der Creative Commons Namensnennung 4.0 International Lizenz veröffentlicht, welche die Nutzung, Vervielfältigung,
Bearbeitung, Verbreitung und Wiedergabe in jeglichem Medium und Format erlaubt, sofern Sie den/die ursprünglichen Autor(en) und die Quelle ordnungsgemäß nennen, einen Link zur Creative Commons Lizenz beifügen und angeben, ob Änderungen vorgenommen wurden.

Die in diesem Artikel enthaltenen Bilder und sonstiges Drittmaterial unterliegen ebenfalls der genannten Creative Commons Lizenz, sofern sich aus der Abbildungslegende nichts anderes ergibt. Sofern das betreffende Material nicht unter der genannten Creative Commons Lizenz steht und die betreffende Handlung nicht nach gesetzlichen Vorschriften erlaubt ist, ist für die oben aufgeführten Weiterverwendungen des Materials die Einwilligung des jeweiligen Rechteinhabers einzuholen.

Weitere Details zur Lizenz entnehmen Sie bitte der Lizenzinformation auf http://creativecommons.org/ licenses/by/4.0/deed.de.

\section{Literatur}

1. Chung JW, Meltzer DO (2009) Estimate of the carbon footprint of the US health care sector. JAMA 302(18):1970-1972

2. Eckelman MJ, Sherman J (2016) Environmental impacts of the U.S. health care system and effects on publichealth. PLoSONE 11(6):e157014

3. Karliner J SS, Boyd R, Ashby B, Steele K (2019) National emmissions snapshots. Health care's climate footprint. ARUP - Health Care Without Harm. September:1-48

4. Watts N, Amann M, Arnell N, Ayeb-Karlsson S, Belesova K, Boykoff M et al (2019) The 2019 report of the Lancet Countdown on Health and Climate Change: ensuring that the health of a child born today is not defined by a changing climate. Lancet 394(10211):1836-1878

5. Vollmer Martin KRTS, Rigby M, Hofstetter D, Hill M, Schoenenberger F, Reimann S (2015) Modern inhalation anesthetics: potent greenhouse gases in the global atmosphere. Geophys Res Lett 42:1606-1611

6. IshizawaY (2011) Special article:general anesthetic gases and the global environment. Anesth Analg 112(1):213-217

7. Luthi D, LeFloch M, Bereiter B, BlunierT, Barnola JM, Siegenthaler U et al (2008) High-resolution carbon dioxide concentration record 650,000-800,000 years before present. Nature 453(7193):379-382

8. (2019) National-Oceanic-and-Atmospheric-Administartion Up-to-date weekly average $\mathrm{CO}_{2}$ at Mauna Loa. https://www.esrl.noaa.gov/gmd/ ccgg/trends/weekly.html/. Zugegriffen: 22. März 2020

9. Brook EJ, Buizert C (2018) Antarctic and global climate history viewed from ice cores. Nature 558(7709):200-208

10. European project for ice coring in antarctica (EPI(A), from May 2001 until April 2004, https://cordis. europa.eu/project/id/EVK2-CT-2000-00077. Zugegriffen: 08.04.2020

11. Masson-Delmotte $\mathrm{V}$, Zhai $\mathrm{P}$, Pörtner $\mathrm{H}-\mathrm{O}$, Roberts D, Skea J, Shukla PR, Pirani A, Péan C, Pidcock R, Connors S, Matthews JBR, Chen Y, Zhou X, Gomis MI, Lonnoy ETM, Tignor M, Waterfield T (2018) Summary for policymakers. In: Global Warming of $1.5^{\circ} \mathrm{C}$. An IPCC Special Report on the impacts of global warming of $1.5^{\circ} \mathrm{C}$ above pre-industrial levels and related global greenhouse gas emission pathways, in the context of strengthening the 
global response to the threat of climate change, sustainable development, and efforts to eradicate poverty. IPCC. 1-24

12. Edenhofer $O F C$, Kalkuhl M, Knopf B, Pahle $M$ (2019) Optionen für eine CO2-Preisreform. MCC-PIK-Expertise für den Sachverständigenrat zur Begutachtung der gesamtwirtschaftlichen Entwicklung. Mercator Research Institute on Global Commons and Climate Change (MCC) gGmbH. Juli:1-106

13. McGain F, Bishop JR, Elliot-Jones LM, Story DA, Imberger GL (2019) A survey of the choice of general anaesthetic agents in Australia and New Zealand. Anaesth Intensive Care 47(3):235-241

14. Ryan SM, Nielsen CJ (2010) Global warming potential of inhaled anesthetics: application to clinical use. Anesth Analg 111(1):92-98

15. Sulbaek Andersen MP, Sander SP, Nielsen OJ, Wagner DS, Sanford TJ Jr., Wallington TJ (2010) Inhalation anaesthetics and climate change. $\mathrm{Br}$ J Anaesth 105(6):760-766

16. Etminan M, Myhre G, Highwood EJ, Shine KP (2016) Radiative forcing of carbon dioxide, methane, and nitrous oxide: a significant revision of the methane radiative forcing. Geophys Res Lett 43(24):12614-12623

17. Sulbaek Andersen Mads PNOJ, Karpichev B, Wallington TJ, Sander SP (2012) Atmospheric chemistry of Isoflurane, Desflurane, and Sevoflurane: kinetics and mechanisms of reactions with chlorine atoms and $\mathrm{OH}$ radicals and global warming potentials.J Phys Chem 116:5806-5820

18. Umwelt Bundesamt (2013) Das Protokoll von Kyoto zum Rahmenübereinkommen der Vereinten Nationen über Klimaänderungen. https:// www.umweltbundesamt.de/themen/klimaenergie/internationale-eu-klimapolitik/kyotoprotokoll\#entstehungsgeschichte-und-ersteverpflichtungsperiode.Zugegriffen: 14.04 .2020

19. Hurwitz MM, Fleming EL, Newman PA, Feng L, Liang Q (2016) Early action on HFCs mitigates future atmospheric change. Environ Res Lett 11:114019

20. Vollmer MK, Rhee TS, Rigby M, Hofstetter D, Hill M, Schoenenberger $F$ et al (2015) Modern inhalation anesthetics: potent greenhouse gases in the global atmosphere. Geophys Res Lett 42(5):1606-1611

21. Gschrey B, Schwarz W, Kimmel T, Zeiger B, Reitz S. Implementierung der ab dem Berichtsjahr 2013gültigen IPCC Guidelines for National Greenhouse Gas Inventories 2006in die Inventarerhebung fluorierter Treibhausgase ( $\mathrm{HFKW}, \mathrm{FKW}_{1}, \mathrm{SF}_{6}, \mathrm{NF}_{3}$ ). Umweltbundesamt: https://www.umweltbundesamt.de/ sites/default/files/medien/378/publikationen/ climate_change_17_2015_implementierung_ der_ab_dem_berichtsjahr_2013.pdf. Zugegriffen:23. März 2020

22. Charlesworth M, Swinton F (2017) Anaesthetic gases, climate change, and sustainable practice. LancetPlanet Health 1(6): e216-e217

23. Sherman J, Le C, Lamers V, Eckelman M (2012) Life cycle greenhouse gas emissions of anesthetic drugs. Anesth Analg 114(5):1086-1090

24. Ozelsel TJ, Sondekoppam RV, Buro K (2019) The future is now-it's time to rethink the application of the global warming potential to anesthesia. Can J Anaesth 66(11):1291-1295

25. Muret J, Fernandes TD, Gerlach H, Imberger G, Jornvall $\mathrm{H}$, Lawson C et al (2019) Environmental impacts of nitrous oxide: no laughing matter! Comment on Br J Anaesth 2019; 122:587-604. Br J Anaesth 123(4):e481-e482

26. Ang TN, Young BR, Taylor M, Burrell R, Aroua MK, Baroutian S (2020) Breakthrough analysis of con- tinuous fixed-bed adsorption of sevoflurane using activated carbons. Chemosphere 239:124839

27. ZeoSys Medical GmbH (2010) Forschung - Narkosegase. https://www.zeosys-medical.de/index. php?page $=$ forschung/narkosegase. Zugegriffen 23. März 2020

28. MacNeill AJ, Lillywhite R, Brown CJ (2017) The impact of surgery on global climate: a carbon footprinting study of operating theatres in three health systems. Lancet Planet Health 1(9):e381-e388

29. Pichler PP, Jaccard IS, Weisz U, Weisz H (2019) International comparison of health care carbon footprints. Environ Res Lett 14(6)

30. Quaschning V (2018) Effizienz und KWK - ein gutes Doppel für den Anfang, 4. Aufl. Carl Hanser, München, S 106-107

31. Dickhoff A (2019) Klik Green - Krankenhaus trifft Klimaschutz. http://klik-krankenhaus.de/ startseite/. Zugegriffen: 23. März 2020 (BUND Deutschland)

32. Wyssusek KH, Keys MT, van Zundert AAJ (2019) Operating room greening initiatives - the old, the new, and the way forward: a narrative review. Waste Manag Res 37(1):3-19

33. Australian-And-New-Zealand-College-Of-Anaesthetists (2017) Climate smart anaesthtists for a better environment and improved health. http:// www.anzca.edu.au/communications/media/ media-releases-2017-(1)/climate-smart-anaesthetists-for-a-better-environme. Zugegriffen: 23. März 2020 (ANZCA)

34. Royal-College-of-Anaesthetists (2018) Environment and sustainalbility. https://www.rcoa.ac. uk/about-college/strategy-vision/environmentsustainability. Zugegriffen: 23. März2020 (RCOA)

35. McGain F, Algie CM, O'Toole J, Lim TF, Mohebbi M Story DA et al (2014) The microbiological and sustainability effects of washing anaesthesia breathing circuits less frequently. Anaesthesia 69(4):337-342

36. Chasseigne V, Leguelinel-Blache G, Nguyen TL, de Tayrac R, Prudhomme M, Kinowski JM et al (2018) Assessing the costs of disposable and reusable supplies wasted during surgeries. Int J Surg 53:18-23

37. Atcheson CL, Spivack J, Williams R, Bryson EO (2016) Preventable drug waste among anesthesia providers: opportunities for efficiency. J Clin Anesth 30:24-32

38. Mankes RF (2012) Propofol wastage in anesthesia. Anesth Analg 114(5):1091-1092

39. McGain F, Story D, Kayak E, Kashima Y, McAlister S (2012) Workplace sustainability: the "cradle to grave" view of what we do. Anesth Analg 114(5):1134-1139

40. Overcash M (2012) A comparison of reusable and disposable perioperative textiles: sustainability state-of-the-art 2012. Anesth Analg 114(5):1055-1066

41. Eckelman M, Mosher M, Gonzalez A, Sherman J (2012) Comparative life cycle assessment of disposable and reusable laryngeal mask airways. Anesth Analg 114(5):1067-1072

42. Sherman JD, Raibley LAT, Eckelman MJ (2018) Life cycle assessment and costing methods for device procurement: comparing reusable and singleuse disposable laryngoscopes. Anesth Analg 127(2):434-443

43. Sherman JD, Hopf HW (2018) Balancing infection control and environmental protection as a matter of patient safety: the case of laryngoscope handles. Anesth Analg 127(2):576-579

44. McGain F, McAlister S, McGavin A, Story D (2010) The financial and environmental costs of reusable and single-use plastic anaesthetic drug trays. Anaesth Intensive Care 38(3):538-544

45. McGain F, McAlister S, McGavin A, Story D (2012) A life cycle assessment of reusable and singleuse central venous catheter insertion kits. Anesth Analg 114(5):1073-1080

46. Hubbard RM, Hayanga JA, Quinlan JJ, Soltez AK, Hayanga HK (2017) Optimizing anesthesia-related waste disposal in the operating room: a brief report. Anesth Analg 125(4):1289-1291

47. Umweltbundesamt (2014) Informationssammlung: Best Practice Municipal Waste Management. https://www.umweltbundesamt. de/informationssammlung-best-practicemunicipal-waste. Zugegriffen: 23. März 2020 (Umweltbundesamt)

48. McGain F, White S, Mossenson S, Kayak E, Story D (2012) A survey of anesthesiologists' views of operating room recycling. Anesth Analg 114(5):1049-1054

49. Goepel M (2016) In: Brauch HG (Hrsg) The great mindshift-how a new economic paradigm and sustainability transformations go hand in hand, 2. Aufl. Springer, Mosbach, S 183

50. Guetter CR, Williams BJ, Slama E, Arrington A, Henry MC, Moller MG et al (2018) Greening the operating room. Am JSurg 216(4):683-688

51. Sorkin AR (2020) BlackRock C.E.O. Larry Fink: Climate Crisis Will Reshape Finance. The New York Times. https://www.nytimes.com/2020/01/14/ business/dealbook/larry-fink-blackrock-climatechange.html.Zugegriffen:23. März 2020

52. Guardian T (2019) UK banks and insurers to be tested on climate crisis response plans-Bank of England to force firms to say how they would respond to temerature rise of up to $4{ }^{\circ} \mathrm{C}$. The Guardian. https://www.theguardian.com/business/ 2019/dec/18/uk-banks-insurers-climate-crisisstress-tests-bank-of-england. Zugegriffen: 23. März 2020

53. Guardian T (2019) Tackling climate crisis is what we should be doing, says new IMF boss-Kristalina Georgieva. The Guardian. https://www.theguardian.com/business/2019/ nov/30/imf-boss-kristalina-georgiva-climatecrisis-financial-crash-economics. Zugegriffen: 23. März 2020

54. Abbasi K, Godlee F (2020) Investing in humanity: The BMJ's divestment campaign. BMJ 368:m167

55. Pressestelle-Ärztekammer-Berlin (2017) Berliner Ärzteversorgung für nachhaltige Kapitalanlagestrategie mit DivestmantAward ausgezeichnet. https://www.aerztekammer-berlin.de/40presse/ 10_Pressemitteilungen/676_DivestmentAward/ index.shtml. Zugegriffen: 23. März 2020 (Ärztekammer Berlin)

56. Law A, Duff D, Saunders P, Middleton J, McCoy D (2018) Medical organisations must divest from fossil fuels. BMJ 363:k5163

57. World_Medical_Association WMA (2019) Resolution on climate emergency. https://www.wma. net/policies-post/wma-resolution-on-climateemergency//.Zugegriffen:23. März 2020 
Hier steht eine Anzeige.

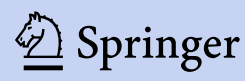

